\title{
A Multi-Agent Model for Assessing Electricity Tariffs
}

\author{
Ioana Pisica, Colin J. Axon, \\ Peter R. Hobson, Gareth A. Taylor \\ School of Engineering and Design \\ Brunel University \\ London, UK \\ Ioana.Pisica@brunel.ac.uk
}

\author{
David C.H. Wallom \\ Oxford e-Research Centre \\ University of Oxford \\ Oxford, UK
}

\begin{abstract}
This paper describes the framework for modelling a multi-agent approach for assessing dynamic pricing of electricity and demand response. It combines and agent-based model with decision-making data, and a standard load-flow model. The multi-agent model described here represents a tool in investigating not only the relation between different dynamic tariffs and consumer load profiles, but also the change in behaviour and impact on low-voltage electricity distribution networks.
\end{abstract}

Index Terms - agent based modelling, electricity tariffs, time of use tariffs

\section{INTRODUCTION}

Demand response programmes communicate with consumers and enable or encourage them to lower or shift consumption. For electricity use, it involves some combination of (a) dynamic pricing, (b) improved feedback of information to the end-users on the amount and timing of their consumption (and generation, if applicable); and (c) some appliance automation such as water heating. Real-time electricity pricing has the potential to smooth out demand to improve network management and reduce emissions where peak demand is met by carbon-intensive generation [1]. In the UK, real-time prices are applied only to large consumers. Residential users contribute significantly to the diurnal peak demand, but as yet there is no compelling argument for involving them in loadshifting. Indeed, reducing load is likely to provide greater environmental benefit [2]. This may change as more distributed generation, and electric vehicles come on stream. The agent-based model (ABM) proposed here was designed to investigate simultaneously (1) consumer behaviour to pricing patterns which develop as a result of near-to-real-time utility use of metering information and (2) the impact on demand on the distribution grid.

The ABM is a dynamic test bed between the human / social systems analysis and the coarse-grained considerations of network operations. The interaction of these agents with the grid can be non-intuitive, and ABM is an appropriate tool to examine these effects. ABMs have been used for other power systems applications such as network control $[3,4]$, protection [5], and wholesale market analysis [6]. Some work has been undertaken to use an $\mathrm{ABM}$ on electricity infrastructure incorporating domestic CHP [4] or heat storage capabilities and emissions reductions, and the trade-off between electricity-led and heat-led paradigms [7]. To the best of our knowledge there are no reported studies which explicitly incorporate network topology.

An understanding of dynamic tariffs and their potential limits of acceptance was developed by using focus groups and interview data to inform the global rules for the ABM from the viewpoint of both electricity producer and consumers in the UK [8]. A general description of the model specification is detailed in Section II. The outcomes of electricity users focus groups were used as direct inputs to the multi-agent model and the methodology is described in Section III. Section IV presents results that validate the implementation of the proposed model and Section V contains the conclusions.

\section{GENERAL FRAMEWORK}

\section{A. Agents definition and goals}

Each agent represents a household that is performing its usual tasks (cooking, washing etc). The inputs are previous bills and past consumption patterns. Agents also have access to tariff ratings from other agents within the same social community. There are two types of goals: conservative and proactive.

The conservative goal is to maintain the life satisfaction level and bill values from epoch to epoch:

$$
\begin{gathered}
\text { Satisfaction }=f(\text { maintain_lifestyle } \& \text { perform_usual_chores) } \\
\qquad \frac{\sum_{i=1}^{n r t} p_{i, k} \cdot W_{i, k}}{\text { satisfaction }_{k}} \leq \frac{\gamma \cdot \sum_{i=1}^{n r t} p_{i, k-1} \cdot W_{i, k-1}}{\lambda \cdot \text { satisfaction }_{k-1}}
\end{gathered}
$$

where $\lambda$ is the willingness to change behaviour, $\gamma$ is the price responsiveness of agent $k$, and $n r t$ is the number of tariff intervals.

The satisfaction is quantified from the lifestyle and the household tasks that are fulfilled. This approach was derived from the outcomes of discussions during focus groups with consumers, that highlighted the importance of being able to maintain their usual behaviour irrespective of electricity tariffs. A tariff that would require shifting loads to avoid bill increases during meals preparation would result in a poor 
satisfaction factor for large families, while for single occupants a shift of this load would not be considered too disruptive [8].

The proactive goal is to minimize the bill value while maintaining life satisfaction:

$$
\min \frac{\gamma \cdot \sum_{i=1}^{n r t} p_{i} \cdot W_{i}}{\lambda \cdot \text { satisfaction }}
$$

Energy consumption can be divided into rigid load (inelastic base load) and elastic demand. Willingness to change behaviour relates to elastic demand.

\section{B. Behavioural classes}

An agent's behaviour is determined by its order of actions and its strategies for achieving the goal. There are two behavioural classes that can be taken into account here: optimization of an objective and learning from experience.

The willingness to change behaviour can be limited by:

- Type of household (large family with children - high inelastic demand).

- Financial status (wealthy and less responsive to price).

- Restricted information (not aware of potential benefits from changing behaviour).

Enablers in changing behaviour:

- Technology adoption (smart house control).

- Policy and regulation.

- Potential financial savings awareness created by:

- Suppliers.

- Media.

- Other agents.

- Environmental awareness education.

\section{Agent specification}

The aim of the modelling framework is to allow the investigation of different tariffs impact on consumers and on power network loading. Each agent can be specified by a set of 14 characteristics:

1. Price responsiveness level of agents: min and max levels is set by the user and the simulation distributes values between these limits to agents in a random manner.

2. Distributed generation: agents can have distributed energy resources with a probability given by the user.

3. Max memory: number of months an agent looks back to compare bills.

4. Agents mobility: how often agents move house and in what proportion.

5. Agent types distribution: category an agent belongs to and in what proportion (single occupancy, family etc.).

6. Trustworthiness: to what degree an information from an agent can be used by other agents.

7. Environmental awareness: informational incentive to reduce demand.

8. Technological acceptance: to what degree an agent adopts new technologies.

9. Financial status: resulting in the price responsiveness (wealthy agents do not minimize the bill value).
10. Percentage of load reduction: min and max percentages with which an agent accepts to reduce its load

11. Number of appliances an agent is willing to time-shift.

12. Size of social community: max number of agents with whom an agent communicates. Each agent has a randomly distributed number of agents it interacts with.

13. Social community resilience: proportion of agents remain in a social community of an agent moving house.

14. Community integration: how many new agents are added to an agent's social community after moving house and after how long.

\section{MODELLING APPROACH}

The role of the proposed model is to provide a tool in assessing different electricity tariff structures and their impact on power network loading. Therefore, the tariff structures to be investigated are presented to a pool of agents connected to a distribution network. The results of the model power flows, voltage levels, loading, losses, and the number of switches to a certain tariff (giving the tariff popularity). The modelling approach is depicted in Figure 1.

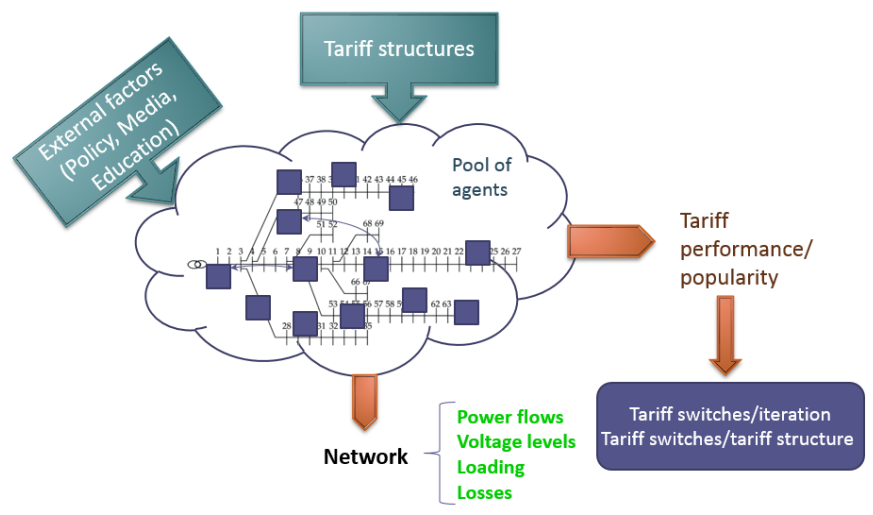

Figure 1. Modelling approach

The agents are based on a template designed to emulate real-life households. This was ensured by using characteristics derived from clustering load profiles of real consumers. The data set used was derived from 197 UK and Bulgarian households [9]. Behaviours for different household-types were identified with cluster analysis using a Dirichlet Process Mixture Model [10] that does not require the number of cluster to be declared a priori. Each agent's load profile is initialized with random values around one of three cluster centroids. Agents are then distributed into an electric power network to make up the load of the network analysed.

The tariff structures that are presented to the pool of agents are specified by electricity prices for each time slot during the day. The time slots dimension can be set from 15 minutes to one hour intervals. Alongside prices, each tariff is specified through a set of non-monetary characteristics that account for user difficulties in adopting, using, or understanding a tariff. These characteristics were determined during focus groups [8] with different types of consumers and different tariff designs inspired from time-of-use tariffs tested by European and North American suppliers. They emulate the involvement of users 
necessary to adopt each tariff. Moreover, the technical skills necessary for understanding and using a tariff are taken into account. The tariff characteristics used were social and technical involvement, and complexity of the structure.

The general ABM routine is presented in Figure 2, where Max represents the total number of agents in the simulated pool. The routine that each agent follows (AGENT) is shown in Figure 3. The parameters affecting the agents' behaviour are set once at the initialization stage.

Each epoch in the model is represented by 24 hours and the simulation time is given by the number of days that the model is run for. Therefore, the agent routine is called Max times for each day simulated. The initialization is run once, at the beginning of the main routine, as showed in Figure 2.

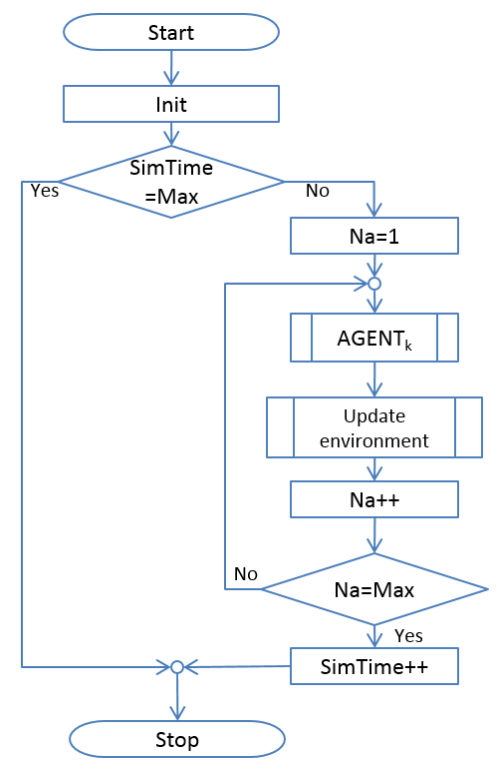

Figure 2. Main ABM routine outline

The second part of the algorithm described in Figure 3 is run for the current simulated day. Several steps presented in Figure 3 are accompanied by a letter (a-i). These indicate where parameters are used and they are described here.

Each day, each agent decides if it will shift load during the current day. This decision is implemented as a random process, based on the parameter (a) load_shift_threshold (0$100 \%$ ), which defines the probability of agents to perform load shift. For example, a setting of $40 \%$ indicates $40 \%$ chance of shifting load each day. If a load shift is to be performed, then the agent decides from where and what load will be displaced. This load is then shifted to a new time slot. The parameters involved in this decision are: (b) Max_shift_time (0..24): maximum duration of the time slot that an agent is willing to shift. Example: 6 hours -> actual shifted time slot duration between 0 and 6 hours; (c) Max_shift_distance (0..24): denotes by how many hours an agent is willing to defer its load. Direction of shift: random (before or after the initial time slot). Example: shift time slot= 17, shift time $=2$, shift distance $=4$, direction:positive $->$ the load curve will be modified by subtracting load_shift_value from initial load curve starting from hour 17 to hour 19 and adding this amount starting from hour 21 to 23 (Figure 4); (d) Max_load_shift_value $(0.100 \%)$ : percentage of power that an agent will be willing to shift. For example: agent power $600 \mathrm{~W}$, max_load_shift_value $=40 \%$-> value to actually shift between 0 and $240 \mathrm{~W}$.

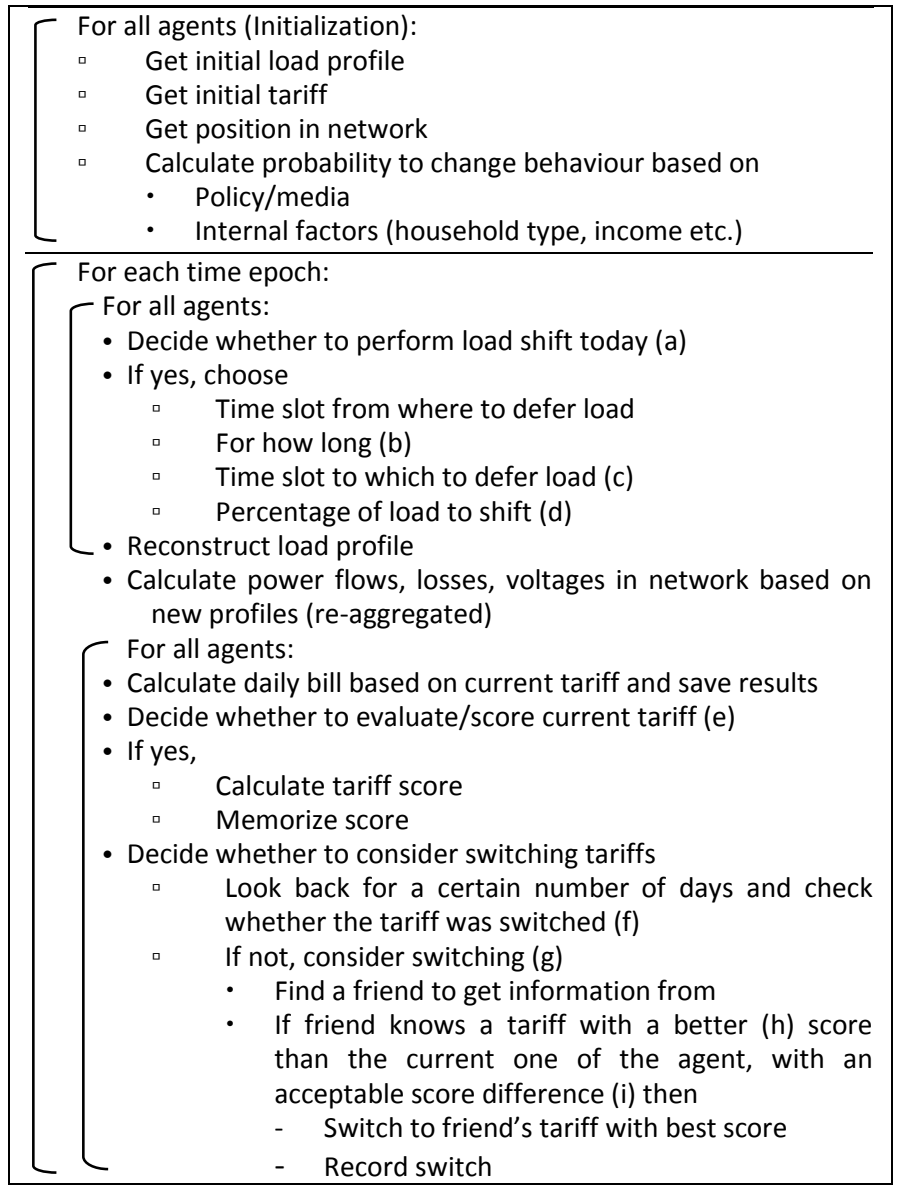

Figure 3. ABM behaviour

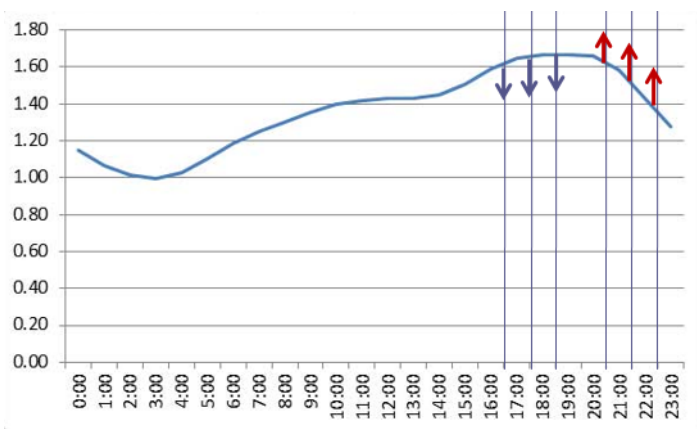

Figure 4. Example of load shifting

Once the load profiles of all agents are reconstructed, the power flow routine is called with the new network loading, resulting in the daily power flows, losses and voltages.

As it is unlikely that real consumers will spend time daily to evaluate their electricity tariffs, a probability to calculate the score of the current tariff at each iteration is specified by parameter (e), Scoring_probability (0-100\%). For example, a scoring probability of $100 \%$ would mean that the tariff is evaluated every day by the agent, calculating the tariff score. 
Thus it is unlikely that agents would switch to different electricity tariffs every day, so the tariff switching is controlled by a set of parameters (f-i). Firstly, it is expected that each agent lets a certain amount of time to pass between two consecutive tariff switches. This is specified for each agent randomly based on parameter (f) Tariff_switch_min_frequency (1-365), representing the minimum number of days that an agent waits until considering switching tariffs. For example: 40 days-> the actual minimum wait time will be a random duration between 40 and 365 days. When an agent exceeds the waiting period, it considers switching based on parameter (g) Switching_probability (0100\%). Finally, based on a Score_difference_threshold (h) and a Tariff_scoring_method (i), the agent chooses a new tariff and switches to it. The Score_difference_threshold (0$100 \%$ ) represents the minimum difference between the agent's own tariff score and the maximum score of another agent's tariff that leads to switching tariff. This controls the sensitivity of agents to tariff scores and emulates the human behaviour to make a change only if the difference would be significant.

The parameters (a) to (i) can be adjusted by the model user allowing investigation of a wide range of agent behaviours and policies that would affect tariff-switching in real life. The values generated inside the routine are based on normal distributions.

\section{Using The Multi-Agent IMPLEMENTATION}

To validate the $\mathrm{ABM}$ implementation several tests were performed with a pool of 10,000 agents. The cluster centroids used for creating the agents profiles are shown in Figure 5, with the resultant load profiles in Figures 6-8.

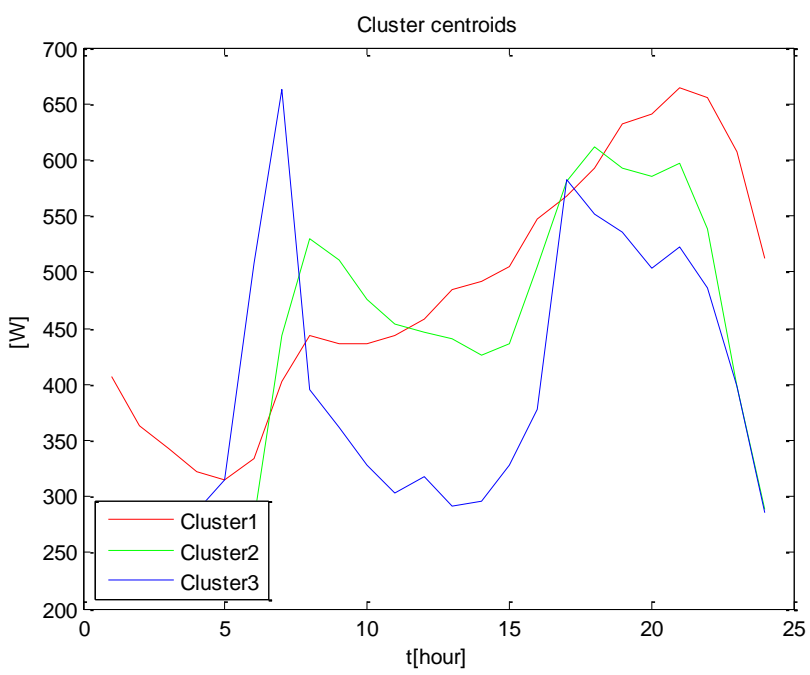

Figure 5. Initial cluster centroids

As the initial dataset was divided into the three clusters in the proportions $36 \%$ (cluster 1), 53\% (cluster 2), and 11\% (cluster 3 ), the agents were created randomly to maintain the same proportions. Figures 6-8 show that the load profiles for the agents are correctly created with normal distributions around the centroids.

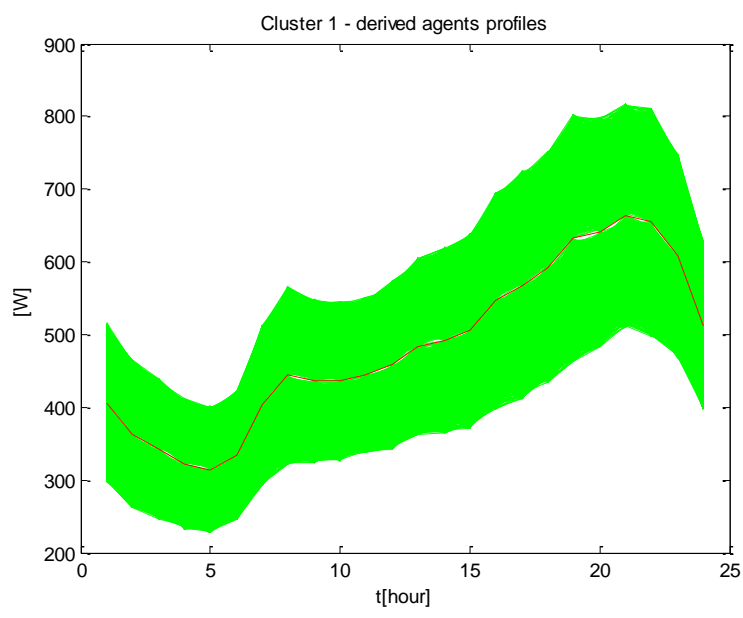

Figure 6. Load profiles of agents created based on Cluster 1 (3600 profiles)

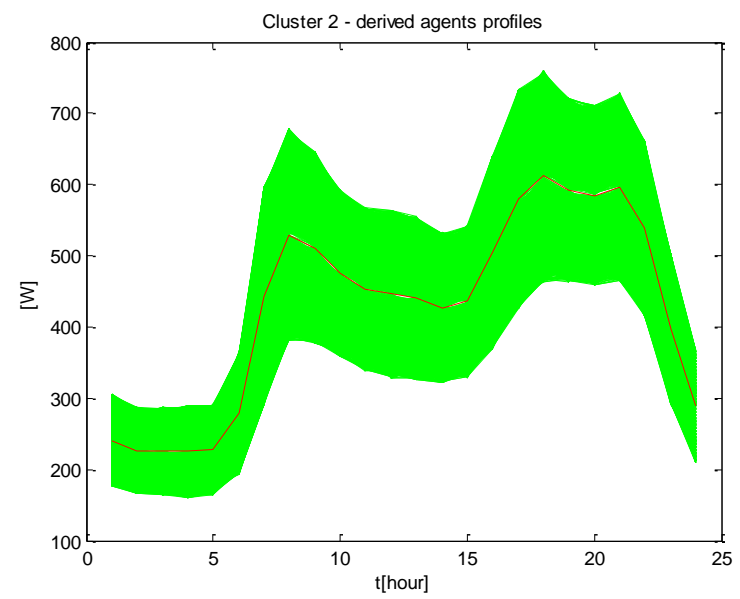

Figure 7. Load profiles of agents created based on Cluster 2 (5280 profiles)

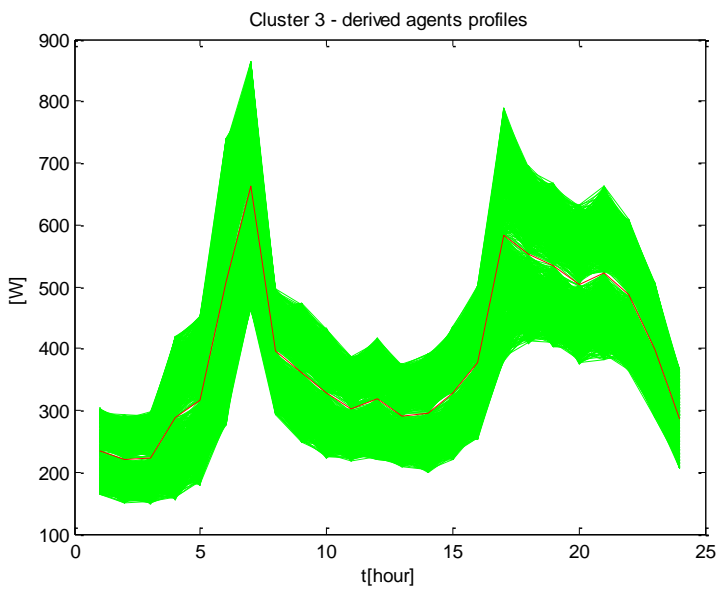

Figure 8. Load profiles of agents created based on Cluster 3 (1120 profiles)

For testing purposes, the 10,000 agents were distributed randomly in the IEEE 69-bus radial network. The load profiles obtained with this setup, during the same simulated day in one of the runs, are presented in figures 9-11. 


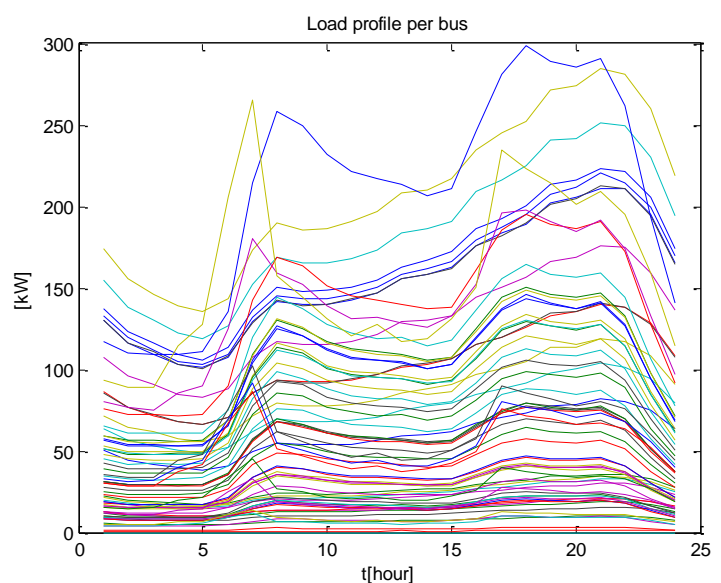

Figure 9. Load profiles for each bus in the network during one simulation day

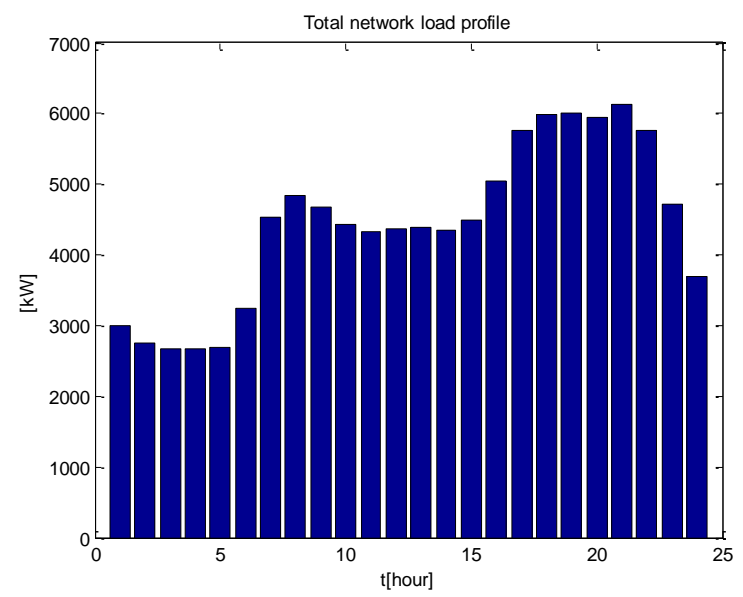

Figure 10. Aggregated bus loading of the network during one simulation day

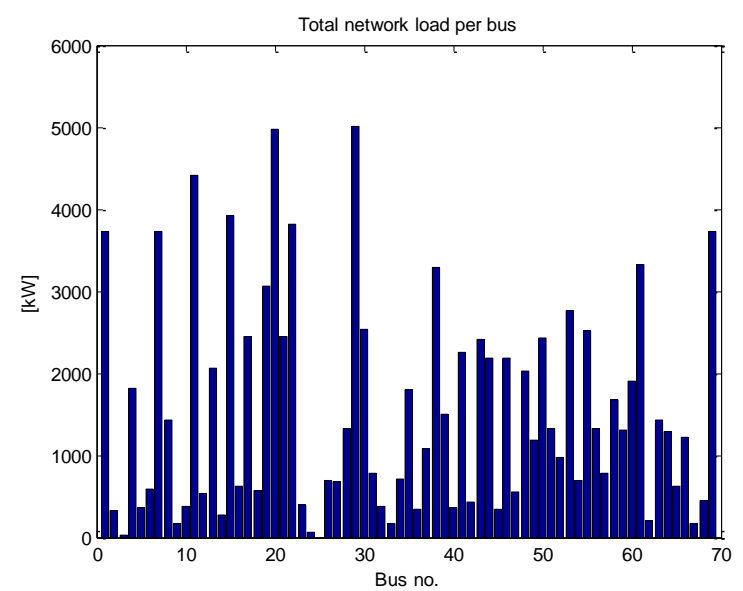

Figure 11. Total loading of the network in each bus

For comparison, the model was run for several parameter values. Two of the setups, where only one parameter differs, are presented here. Table I shows the values used for the parameters during the two runs. Figures 12 and 13 present results for the first setup and Figures 14 and 15 for the second setup.
The simulation time is set to one year (365 days) in both cases. The only parameter that is varied is the switching probability. The agents are presented three tariff structures, with complexities of 40,80 and $65 \%$ respectively. The results show that for the same tariff structures presented to the agents, the number of switches for each of the tariffs is different during the two runs. Figures 12 and 14 show that no switches are performed in the first 60 days of the simulation, as expected from the parameter tariff_switch_min_freq value.

Table I. Parameter setting

\begin{tabular}{|c|c|c|}
\hline Parameter name & Setup1 & Setup2 \\
\hline load_shift_threshold [\%] & 50 & 50 \\
\hline max_load_shift_value [\%] & 40 & 40 \\
\hline max_shift_time [0-24] & 4 & 4 \\
\hline max_shift_distance [0-24] & 6 & 6 \\
\hline scoring_probability [\%] & 60 & 60 \\
\hline tariff_switch_min_freq [1-365] & 60 & 60 \\
\hline switching_probability [\%] & $\mathbf{3 0}$ & $\mathbf{1 0}$ \\
\hline score_difference_threshold [\%] & 10 & 10 \\
\hline sim_time [no of days] & 365 & 365 \\
\hline tariff_scoring_method (min,max) & 1 & 1 \\
\hline
\end{tabular}

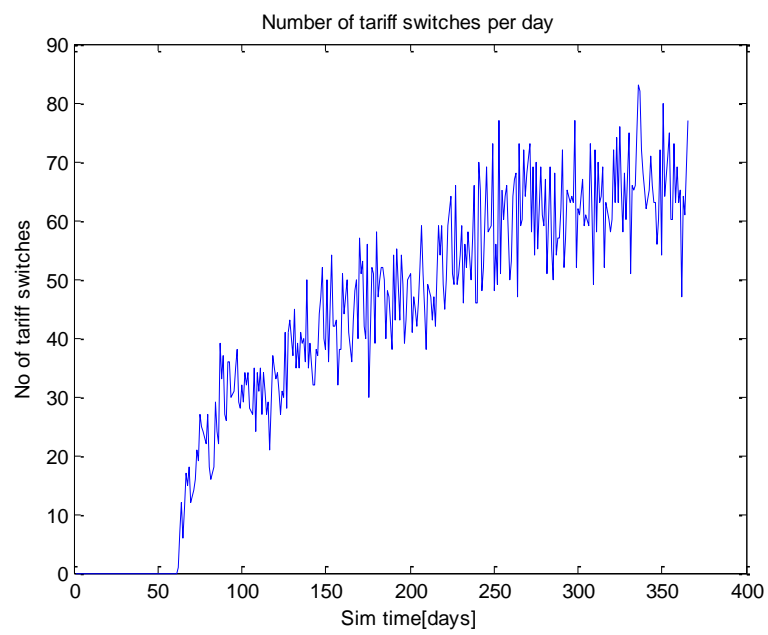

Figure 12. Number of tariff switches each day of simulation - setup1

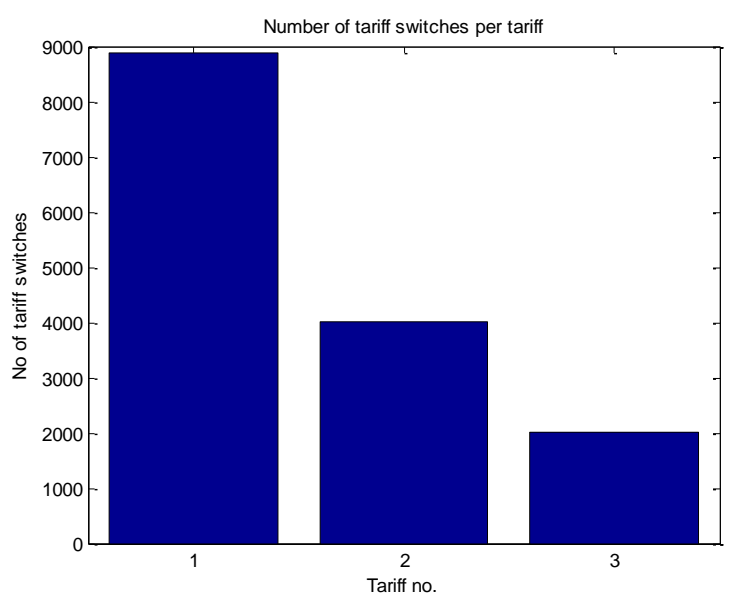

Figure 13. Number of witches to each tariff - setup1 


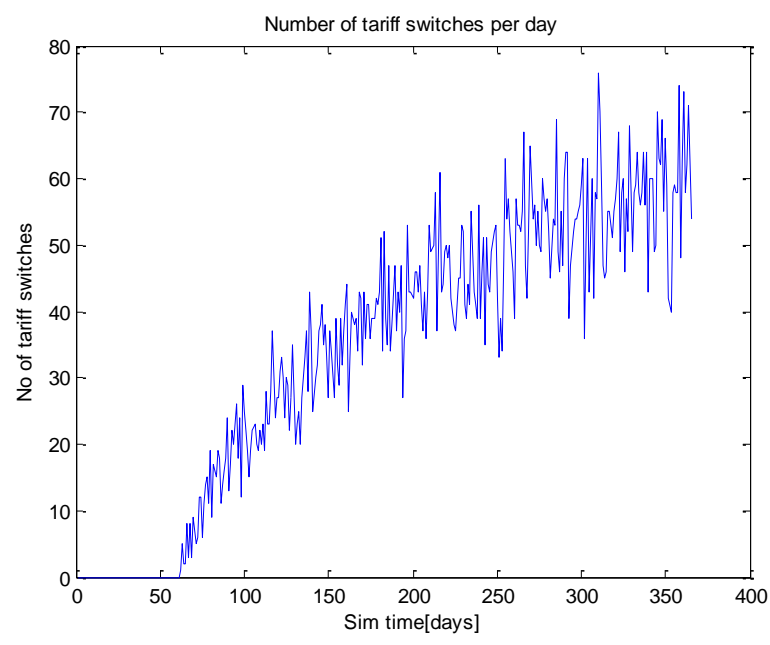

Figure 14. Number of tariff switches each day of simulation - setup2

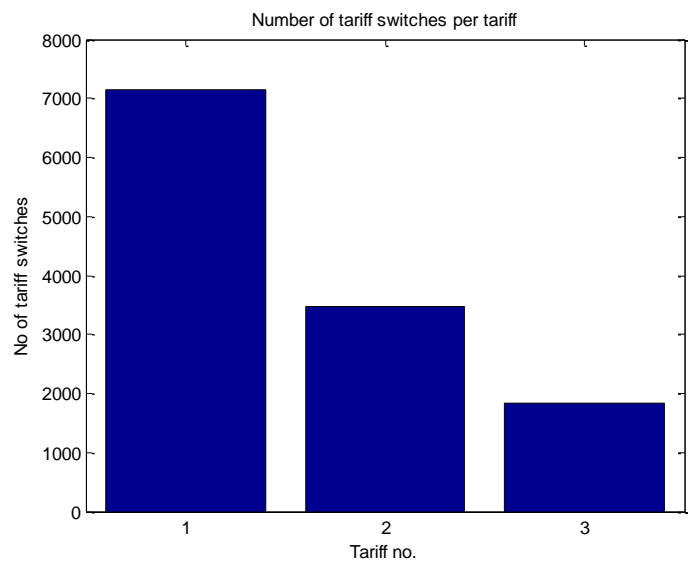

Figure 15. Number of witches to each tariff - setup2

\section{CONLCUSIONS}

Demand side response can facilitate improved network management and reduce costs across the electricity system by allowing investment in new capacity to be deferred. One tool to enable DSR is time varying tariffs - whether time-of-use or dynamic with near-to-real-time pricing. The role of demand side response is likely to increase as distributed generation and new loads such as electric vehicles and heat pumps. The new loads will, if left unchecked, increase the size of daily demand peaks. Distributed generation will increase the complexity of network management. In both cases, new tariffs reacting to retail market conditions may inadvertently create perverse incentives or unexpected outcomes.

To this end, an ABM is a flexible tool to investigate potential effects on the low-voltage distribution network. Static time of use tariffs which reflect the underlying electricity system costs by time of day, can help reduce system costs by providing incentives to customers to shift demand to times when system costs are lower.

This paper investigated dynamic electricity tariffs by modeling a multi-agent framework. The agents and interactions are designed, implemented and tested and the results are presented to validate the proposed model.

This tool can be used by both social scientists and those looking into the technical implications of electricity tariffs by allowing the user to specify a wide range of parameters that help in emulating households and their behavior in relation with load shifting patterns.

Future work can focus on several questions, including:

- Social community size - how many friends to take into account before switching.

- Use the type of household and load factor to determine flexibility instead of shifting load randomly.

- Different network topologies.

- Include distributed generation - agent profiles changing depending on weather.

- Take into account communication issues. What happens if at some point an agent does not receive tariff information?

\section{ACKNOWLEDGMENT}

The authors acknowledge the contribution of the EPSRC Transforming Energy Demand Through Digital Innovation Programme, grant agreement numbers EP/I000194/1 and EP/I000119/1, to the ADEPT project.

\section{REFERENCES}

[1] Axon, CJ, Darby, SJ, Granell, R, Hobson, PR, Layberry, RL, Pisica, I, Taylor, GA, Wallom, DCH (2012) "Towards an understanding of dynamic energy pricing and tariffs", $47^{\text {th }}$ Universities Power Engineering Conference (UPEC), London, 4-7 Sept. 2012.

[2] Shaw R, Atree M, Jackson T and Kay M (2009), 'The value of reducing distribution losses by domestic load-shifting; a network perspective'. Energy Policy, 37, 3159-3167.

[3] Dimeas AL, and Hatziargyriou ND (2005), 'Operation of a Multiagent System for Microgrid Control', IEEE Trans. Power Systems, 20, 3, pp1447-1455.

[4] Koen H van Dam, et al, (2006), 'Modelling an Electricity Infrastructure as a Multi-Agent System - Lessons Learnt from Manufacturing Control', 9th Intl Symp Proc Sys Engng, pp1741-1746.

[5] Wan H, et al, (2006), 'Multi-agent application of substation protection coordination with distributed generators', Euro Trans Electrical Power, 16,5 , pp495-506.

[6] Weidlich A, and Veit D (2008), 'A critical survey of agent-based wholesale electricity market models', Energy Economics, 30, 4, pp1728-1759.

[7] Houwing M and Bouwmans I (2006), 'Agent-based modelling of residential energy generation with micro-CHP', $2^{\text {nd }}$ Intl Conf on Integration of Renewables and Distributed Energy Resources.

[8] Darby, SJ, and Pisica, I (2013), 'Focus on electricity tariffs: experience and exploration of different charging schemes'. Paper 8-318-13, European Council for an Energy-Efficient Economy summer study, Hyères, June 3-7, 2013.

[9] Sowden, R, and Tommis, M (2011), 'D7.7 project cycle analysis report for cycle 3', project Dehems, www.dehems.eu.

[10] Granell, R, Axon, CJ, and Wallom, DCH (2013), 'Dirichlet Process Mixture Model for Clustering Electricity Load Profiles', 9 ${ }^{\text {th }}$ International Conference on Machine Learning and Data Mining (MLDM), 19-25 July, New York City USA, in Machine Learning and Data Mining in Pattern Recognition Poster Proceedings, Ed. Petra Perner, pp.1-6, IBAI, Berlin, Germany. 